\title{
Appareil
}

\section{La guitare électrique augmentée : cadre de travail et stratégies pour la conception d'un instrument augmenté}

\section{Otso Lähdeoja}

\section{(2) OpenEdition \\ Journals}

Édition électronique

URL : http://journals.openedition.org/appareil/1016

DOI : 10.4000/appareil.1016

ISSN : 2101-0714

Éditeur

MSH Paris Nord

Référence électronique

Otso Lähdeoja, « La guitare électrique augmentée : cadre de travail et stratégies pour la conception d'un instrument augmenté », Appareil [En ligne], 5 | 2010, mis en ligne le 10 juin 2010, consulté le 30 juillet 2020. URL : http://journals.openedition.org/appareil/1016 ; DOI : https://doi.org/10.4000/ appareil.1016

Ce document a été généré automatiquement le 30 juillet 2020.

\section{cc)}

Appareil est mis à disposition selon les termes de la Licence Creative Commons Attribution - Pas d'Utilisation Commerciale - Pas de Modification 4.0 International. 


\title{
La guitare électrique augmentée : cadre de travail et stratégies pour la conception d'un instrument augmenté
}

\author{
Otso Lähdeoja
}

\section{Introduction}

1 La guitare électrique constitue un cas particulier dans l'instrumentarium occidental par sa nature d'hybride technologique. Née d'une alliance entre la technologie électromécanique et la lutherie acoustique, son potentiel sonore ne peut s'exprimer pleinement que couplé à un amplificateur et, éventuellement, aux traitements sonores ou « effets ». Après plus de soixante-dix ans de développement et de pratique par une large communauté de musiciens, la guitare électrique présente l'exemple singulier d'un instrument électroacoustique, où des questions de live-electronic comme le contrôle des traitements, la multi-temporalité ${ }^{1}$ ou le lien entre le geste et le son ont pu être expérimentés en pratique dans de multiples contextes musicaux.

2 L'augmentation d'un instrument de musique désigne un processus d'élargissement des capacités d'un instrument traditionnel par des moyens technologiques, sans entraver le fonctionnement de l'instrument initial (Miranda, 2006). Dans ce sens, la guitare électrique est pleinement un instrument augmenté : une guitare acoustique équipée de microphones, de traitements du signal et d'amplification qui en décuplent les possibilités sonores. Toutefois, à l'opposé de certains instruments augmentés plus récents axés sur la question du contrôle timbral en temps réel (Aimi, 2007 ; Machover), la guitare électrique présente un cas où les grandes possibilités sonores ouvertes par l'augmentation n'ont été que peu suivies par le développement de moyens de les contrôler en cours du jeu. Les interfaces et accès gestuels employés habituellement par les guitaristes - potentiomètres, boutons, pédales - sont d'une grande simplicité, sans 
commune mesure avec la sensibilité haptique que requiert le jeu de la guitare ellemême. L'utilisation de la guitare électrique est souvent symptomatique de ce manque d'interaction dynamique avec la partie augmentée de l'instrument, il se caractérise par une utilisation «monolithique » des timbres, sélectionnés par des effecteurs « on-off ", restant statiques pendant toute une partie musicale. Le potentiel sonore et expressif de la guitare électrique n'est ainsi que partiellement exploité, faute de moyens de contrôle, (Lähdeoja, 2008)

3 Le projet de guitare électrique augmentée développé au Centre de recherche et création en informatique musicale (CICM) est né de la confrontation continue de l'auteur à ce problème de contrôle. Jouant de la guitare électrique dans un contexte de création live-electronic, j'ai été frustré pendant des années par l'inadéquation entre la richesse des sonorités que je pouvais obtenir avec des diverses unités de traitement de son, et mon incapacité de les intégrer pleinement dans mon jeu de guitare. La quantité de paramètres à gérer en « live » dépassait de loin ce que je pouvais contrôler avec les potentiomètres et pédales. Les sonorités et le type de jeu que je souhaitais obtenir restaient inaccessibles derrière la barrière des interfaces inadéquates.

\section{La guitare électrique augmentée}

4 Le projet de guitare électrique augmentée entrepris explore les possibilités et les manières de créer des liens entre le corps du guitariste et les traitements audionumériques, afin d'intégrer la dimension numérique à l'environnement instrumental de la guitare d'une manière cohérente, souple et sensible. Ce questionnement implique trois domaines de travail distincts :

5 1) Le domaine du corps, du geste instrumental

6 2) L'acquisition des données gestuelles; techniques, technologies et stratégies de captation

7 3) Le domaine du son ; traitements audionumériques et techniques de synthèse

8 Notre travail de conception des augmentations a pour but de trouver des solutions qui relient ces trois domaines dans un continuum allant du geste vers le son. L'aspect sensible et qualitatif des applications est de première importance dans ce projet. Le vécu et le ressenti de l'instrumentiste est un critère clé dans la validation (ou non) des pistes de recherche.

9 Considérons les trois domaines de recherche séparément :

\section{1. Le geste instrumental de la guitare électrique}

10 Lors du jeu d'un instrument, le corps manipule l'objet avec un répertoire de mouvements très précisément défini : c'est la «technique instrumentale ». Chaque partie du corps connectée à l'instrument a son propre vocabulaire gestuel adapté à sa tâche et aux contraintes de l'objet. Dans le cas de la guitare, à partir d'une technique instrumentale "standard", l'on peut répertorier un ensemble de constituants de ce vocabulaire manuel. Chaque main a une zone de travail spécifique.

11 1) La main gauche travaille sur le manche. Son répertoire de gestes comprend:

- appuyer, combinaisons des quatre doigts sur la matrice définie par les cordes et les frettes ;

- ibrato vertical et horizontal ; 
- légato (hammer-on et pull-off);

- glissé.

2) La main droite pince les cordes avec les doigts ou avec un médiateur. Elle peut également intervenir sur le corps de la guitare ou sur la touche :

- pincer une ou plusieurs cordes ;

- strumming (avec les doigts ou avec le médiateur);

- frapper (cordes ou caisse) ;

- appuis véloces sur la touche, «tapping »;

- pincer en bloquant la corde (harmoniques main droite).

Ces gestes forment la technique de jeu de la guitare. L'on peut être surpris par leur nombre apparemment peu élevé, mais il faut noter que chaque geste comporte en soi ses propres «dimensions » telles que la force ou la localisation précise, et qu'ils se combinent dans le jeu.

Lors du passage à la guitare électrique le répertoire gestuel de la main droite s'étend : tourner un potentiomètre (volume, brillance), actionner un interrupteur (choix de microphone), appuyer sur la barre de vibrato. Aussi, avec l'utilisation de pédales d'effets, un nouveau geste impliquant le pied du guitariste est nécessaire, avec un répertoire de mouvements lié à la nature de la pédale (commutateur «on/off» ou pédale d'expression). L'étendue de la technique instrumentale s'accroît parallèlement à l'augmentation de l'instrument.

15 Au-delà de la technique instrumentale stricte, le corps du musicien effectue divers mouvements qu'on peut classer selon la typologie suivante (Refsum-Jensenius, 2009) :

- gestes effectifs ; production, maintien et modification du son;

- gestes auxilliaires (ancillary gestures); accompagnent les gestes effectifs, auxquels ils sont physiologiquement liés;

- gestes accompagnateurs ; mouvements du corps musicalement engagés, mais sans lien direct avec la production de son;

- gestes communicatifs ; entre les musiciens, du musicien vers le public.

Dans le cadre de l'augmentation d'instruments, diverses stratégies peuvent être adoptées pour utiliser ces catégories du geste. L'on peut «activer» des gestes qui ne participent pas à la production du son en les captant et en leur attribuant une correspondance sonore. L'on peut également utiliser les gestes effectifs, donc associer la partie augmentée de l'instrument avec sa technique de jeu acoustique. Une autre stratégie serait d'utiliser des gestes auxiliaires qui sont subordonnés aux gestes effectifs et qui ne peuvent pas être contrôlés indépendamment de la production sonore. Dans chaque cas, les particularités de la catégorie de geste utilisée influent sur le rapport geste-son, sur l'indépendance que l'augmentation peut avoir par rapport au jeu de l'instrument initial, ainsi que sur le degré de précision du contrôle gestuel (ou degré de « conscience » qu'en a le musicien).

Le corps qui se lie à l'instrument et se meut avec la musique constitue le cadre dans lequel la "greffe technologique» de l'augmentation doit trouver une intégration harmonieuse, sans gêner la technique instrumentale, et sans sur-solliciter diverses parties du corps pour des tâches simultanées demandant de la précision. Domaine éminemment sensible, l'environnement de jeu du musicien pose des contraintes lourdes à tout projet d'augmentation. 


\section{2. L'acquisition des données gestuelles ; techniques, technologies et stratégies de captation}

Afin de pouvoir étendre son contrôle aux traitements audionumériques, le guitariste a besoin de voies d'accès au niveau numérique de l'instrument augmenté. Pour un contrôle basé sur les mouvements du corps, il est nécessaire d'acquérir des données à partir de ses gestes. Dans le cas de la guitare, deux stratégies d'acquisition de données sont possibles (Wanderley, 2001) :

- La captation directe : des variables de grandeurs physiques sont captées avec des appareils de mesure (capteurs).

- La captation indirecte : des données relatives aux gestes de l'instrumentiste sont extraites par l'analyse du son de l'instrument. Sur un instrument acoustique, par transduction énergétique, le son est une « image » du geste qui l'a produit.

Les possibilités de la captation directe découlent des diverses technologies de mesure disponibles et accessibles (par leur prix notamment), et de leur intégration dans l'environnement de jeu de la guitare. Une grande variété de capteurs existe, offrant de multiples possibilités dont notre projet n'a exploré qu'une petite partie. Notre utilisation des capteurs a d'abord été motivée par la recherche de mouvements «disponibles» dans l'environnement de jeu du guitariste: gestes consciemment contrôlables et présentant des degrés de liberté disponibles pendant le jeu. Un travail conséquent a été mené sur l'utilisation des mouvements "auxilliaires" (ancillary gestures) aux gestes liés aux mouvements de la technique instrumentale qui ne sont pas entièrement indépendants et consciemment contrôlés (Lähdeoja, Wanderley, Malloch, 2009).

20 La captation indirecte peut fournir des données utiles concernant les gestes effectifs sur l'instrument. Diverses techniques d'analyse du signal peuvent être utilisées, fournissant des informations sur les attaques, le timbre, la fréquence, la polyphonie etc. des notes jouées: suivi d'enveloppe, analyse spectrale (structure et répartition d'énergie dans le spectre, harmonicité/inharmonicité, centre de gravité, changements brusques du spectre). Dans notre projet, les données obtenues par la captation indirecte sont utilisées à la fois pour produire des effets auto-adaptatifs (Verfaille, 2003), ainsi que pour recueillir des méta-informations du jeu du guitariste servant à contrôler des synthétiseurs et des boucleurs.

21 Une piste intéressante nous semble être l'utilisation simultanée des deux formes de captation disponibles. Dans ce type d'application, la captation indirecte fournit des données relatives au jeu, permettant d'actionner des traitements par le jeu lui-même, tandis que l'évolution des "effets" dans le temps est effectuée par des gestes de contrôle, captés par des appareils de mesure.

L'acquisition des données dans le cadre d'un instrument augmenté est tributaire d'un double cahier des charges. D'abord, la contrainte technologique délimite l'aire des possibilités par les types de capteurs disponibles et les algorithmes d'analyse du signal. En deuxième lieu, l'instrument et le corps du guitariste définissent un environnement physique qui régit sur la possibilité d'utilisation de ces technologies.

23 En se portant sur la guitare électrique, nous pouvons tenter de représenter de manière synthétique les différentes stratégies d'acquisition de données à partir de l'instrument. 
Le tableau ci-dessous présente un ensemble de possibilités. La dimension du geste question particulièrement complexe - n’y est pas développée.

Tableau 1 : stratégies d'acquisition de données de la guitare électrique

\begin{tabular}{|c|c|c|c|}
\hline SOURCE & DESCRIPTION & DONNÉES & NOTES \\
\hline $\begin{array}{l}\text { Microphone } \\
\text { électromagnétique }\end{array}$ & $\begin{array}{l}\text { La sortie audio } \\
\text { «normale » de la G.E. }\end{array}$ & $\begin{array}{l}\text { Signal audio: } \\
\text { spectre, } \\
\text { amplitude } \\
\text { (fréquence, } \\
\text { centroïde, amp } \\
\text { enveloppe, } \\
\text { attaque...) }\end{array}$ & $\begin{array}{l}\text { Analyse du signal biaisé par } \\
\text { la coloration } \\
\text { microphone du } \\
\text { monophonie } \text { et }\end{array}$ \\
\hline $\begin{array}{l}\text { Microphone contact } \\
\text { (ou capteur piezo) }\end{array}$ & $\begin{array}{l}\text { Attachés au corps de la } \\
\text { G.E. fournissent r un } \\
\text { domaine r sonore } \\
\text { percussif, bruits } \quad \text { de } \\
\text { manipulation, golpe etc... }\end{array}$ & $\begin{array}{l}\text { Signal audio } \\
\text { «percussif » }\end{array}$ & $\begin{array}{l}\text { Une «altérité » percussive } \\
\text { intéressante. Bois, métaux } \\
\text { et localisations donnent } \\
\text { des sons et spectres } \\
\text { différents. }\end{array}$ \\
\hline Capteurs & $\begin{array}{lrr}\text { Inclinaison, } & \text { accélération, } \\
\text { pression, } & \text { surface } \\
\text { sensible } & \text { au } & \text { toucher, } \\
\text { flexion... } & \text { Matrice } & 2 \\
\text { valeurs } & \text { sensibles } & \text { au } \\
\text { toucher... } & & \end{array}$ & $\begin{array}{l}\text { Données } \\
\text { contrôle }\end{array}$ & 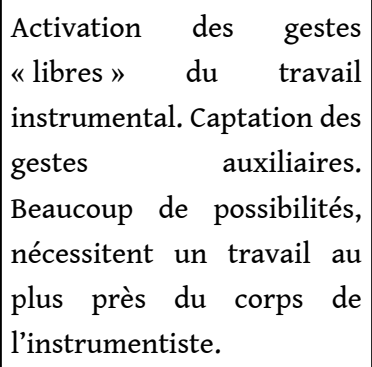 \\
\hline $\begin{array}{l}\text { Contrôleurs } \\
\text { (traditionnels de } \\
\text { l'environnement de jeu } \\
\text { de la guitare } \\
\text { électrique) }\end{array}$ & $\begin{array}{l}\text { Pédale (expression, } \\
\text { commutateur), } \\
\text { potentiomètres et } \\
\text { «boutons on-off » }\end{array}$ & $\begin{array}{l}\text { Données } \\
\text { contrôle }\end{array}$ & $\begin{array}{l}\text { Solutions acceptables, à } \\
\text { intégrer dans un contexte } \\
\text { augmenté }\end{array}$ \\
\hline $\begin{array}{l}\text { Microphone } \\
\text { hexaphonique } \\
\text { (optique, } \\
\text { électromagnétique, } \\
\text { piezo) }\end{array}$ & $\begin{array}{l}\text { Sortie audio de chaque } \\
\text { corde }\end{array}$ & $\begin{array}{lr}\text { Données } & \text { du } \\
\text { signal audio pour } \\
\text { chaque rerde. } \\
\text { Ouvre ra } \\
\text { possibilité } & \text { de } \\
\text { l'analyse } & \\
\text { polyphonique }\end{array}$ & $\begin{array}{l}\text { Grande quantité de } \\
\text { données et calculs lourds } \\
\text { (6fft en permanence). } \\
\text { Beaucoup de possibilités } \\
\text { pour la captation des gestes } \\
\text { dans leur détail. }\end{array}$ \\
\hline $\begin{array}{l}\text { Système guitare MIDI } \\
\text { (Microphone } \\
\text { hexaphonique, } \\
\text { conversion pitch to MIDI) }\end{array}$ & $\begin{array}{l}\text { Sortie audio de chaque } \\
\text { corde, converti en } \\
\text { données MIDI }\end{array}$ & $\begin{array}{l}\begin{array}{l}\text { Données } \\
\text { Noter } \\
\text { velocity, }\end{array} \\
\text { aftertouch, pitch), } \\
\text { bend }\end{array}$ & $\begin{array}{l}\text { Données } \\
\text { exploitables }\end{array}$ \\
\hline
\end{tabular}




\section{3. Le domaine du son; traitements audionumériques et techniques de synthèse} Sensor ayant l'avantage de produire peu d'interférences électromagnétiques avec les signaux de capteurs situés à leur proximité. Sur cette guitare sont montés un capteur d'inclinaison deux axes, une surface sensible au toucher, ainsi qu'un microphone de contact. L'interface de contrôle se prolonge sur le corps du guitariste par un capteur de flexion attaché à son poignet droit, ainsi que par terre par un pédalier comportant deux pédales d'expression et des pédales commutateurs MIDI.

\footnotetext{
- 2 sources audio (sortie mono de la guitare et un microphone de contact) ;
} 
- 4 canaux de données de capteurs (convertis en MIDI) ;

- 2 canaux de données des pédales d'expression ;

- des messages control change du pédalier.

Tous ces flux sont regroupés dans un patch Max/MSP qui procède à l'analyse des données et au traitement et à la synthèse audio. La sortie du système est en stéréo. L'amplificateur de la guitare électrique est remplacé par un logiciel simulant divers types d'amplificateurs et d'effets, servant à la création du timbre initial de la guitare électrique.

Figure 1 : schéma de la guitare électrique augmentée
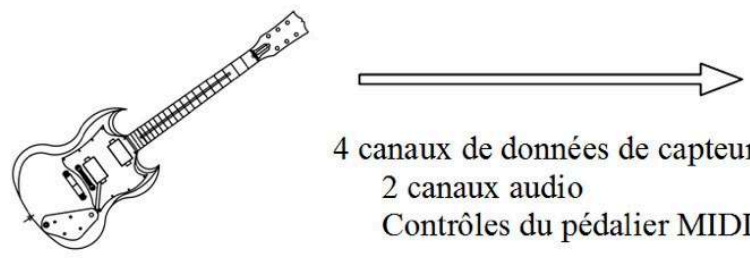

4 canaux de données de capteurs 2 canaux audio Contrôles du pédalier MIDI
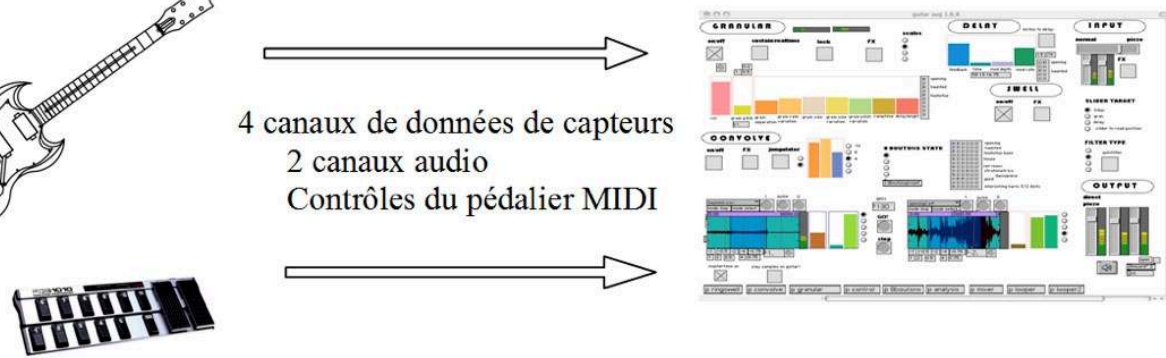

Patch Max/MSP d'analyse et de traitement/synthèse

Le programme Max/MSP comporte les modules sonores suivantes:

- granulateur temps réel, réactualisant sa mémoire interne à chaque note détectée par l'analyse du signal ;

- module de synthèse croisée pour produire des sons percussifs à partir du signal du microphone de contact et d'une banque de samples pré-enregistrée ;

- modulateur en anneau « auto-adaptatif » (ring modulator swell);

$\cdot 2$ boucleurs avec vitesse et sens de lecture contrôlables ;

- section de filtrage ;

- delay ;

- simulateur d'amplificateur.

La mise en correspondance des signaux de contrôle avec les modules sonores a été construite à partir d'un travail "d'incorporation» de nouveaux gestes dans l'environnement instrumental. L'angle frontal de maintien de la guitare influe sur la présence de la granulation dans le mix de sortie, l'angle sagittal contrôle la présence de la modulation en anneau. La surface sensible, ainsi que le capteur de flexion du poignet contrôlent des filtres du son de la guitare et des boucleurs. Les pédales d'expression influent sur le volume d'entrée ainsi que sur le comportement du delay. Les commutateurs de la pédale permettent de (des)activer des parties de l'instrument, comme le mode " percussif » (entrée et traitement du signal piezo), ou de passer d'un état de patch à un autre (presets).

\section{La guitare électrique augmentée en pratique musicale - retour sur deux années d'expérimentation}

La guitare électrique augmentée a été expérimentée en pratique de live electronic depuis fin $2007 \mathrm{au}$ sein du projet $O A O$ combinant musique, projections vidéo et scénographie. 
Le projet a produit une performance jouée une dizaine de fois en public en France et en Angleterre, un DVD, ainsi qu'un disque audio actuellement en mixage. Cette expérience de la pratique de la guitare électrique augmentée semble suffisante pour tenter une première lecture de sa fonctionnalité réelle en tant qu'instrument du live, de ses points forts et faibles.

En premier lieu, les possibilités sonores des techniques audionumériques employées agrandissent considérablement la palette d'expression de la guitare. Les textures granulaires, sons percussifs, delays et filtrages taillés sur mesure sont d'une grande richesse. La création d'un répertoire a été stimulante et gratifiante. Aussi, les nouveaux moyens de contrôle permettent un dynamisme sonore des traitements jusqu'alors inaccessibles pour la guitare électrique traditionnelle, comme la gestion des masses sonores, ou le contrôle des traitements directement par le jeu.

Toutefois un nombre d'inconvénients et de problématiques persiste, dont le plus criant est le contrôle simultané des différentes strates instrumentales, logicielles et sonores. L'environnement augmenté se présente au musicien comme un environnement d'une grande complexité, le poussant à la limite (et parfois au-delà) de ses capacités de gestion. Deux conséquences découlent de cette problématique: 1) la fixation de la musique en formes définies correspondant à une écriture du parcours à suivre dans l'instrument augmenté. Il m'a été très difficile d'improviser d'une manière fluide avec le dispositif, le temps de réponse pour accéder à certaines sonorités ou fonctionnalités semble trop long. Peu à peu les pièces se sont figées selon des états prédéterminés du logiciel. 2) Une certaine lenteur dans la manipulation des matériaux, poussant vers une esthétique des durées et des masses sonores. Il semble difficile d'articuler les sonorités produites aussi rapidement que sur la guitare traditionnelle.

Il faut aussi mentionner la contrainte physique créée par le dispositif. Un ensemble de câbles relie le musicien aux capteurs, pédales, interface audio et ordinateur. Son regard est souvent occupé par la lecture de l'écran. Les mouvements "activés » par les capteurs l'empêchent d'effectuer des mouvements expressifs habituels. Sur scène, le vécu du fait d'être captif du dispositif peut s'avérer frustrant autant pour le musicien que pour le public, par une stabilité, voire une rigidité, corporelle qui diminue l'expressivité physique et l'auto-stimulation par le mouvement. Dans mon expérience, cette sensation m'empêche de jouer au maximum de mes capacités instrumentales, j'ai l'impression d'avoir des difficultés "d'entrer dans la musique " plus qu'avec une guitare traditionnelle.

Une réponse à ces difficultés semble consister dans le développement minutieux de l'ergonomie de l'environnement instrumental augmenté. La création d'une sensation de fluidité et d'unité dans l'instrument augmenté requiert un travail très détaillé d' « accordage » entre les gestes et leurs correspondants sonores. Ce processus passe nécessairement par la pratique de l'instrument, et il permet graduellement d'effacer l'interface au profit d'une connexion directe entre geste et son.

\section{Conclusion et travaux futurs}

38 Le projet de guitare électrique augmentée présenté dans cet article est un travail interdisciplinaire, au carrefour des recherches en musicologie et création musicale, de la technologie, et de la théorie du geste instrumental. Il a été conçu comme un dialogue entre les différentes parties de l'ensemble, ainsi que comme un aller-retour entre la 
théorie et la mise en pratique technologique et musicale. Développé depuis 2007, le projet atteint désormais un stade de relative maturité qui permet d'évaluer les résultats du travail, les acquis ainsi que les zones de problématiques persistantes. La guitare électrique augmentée met en œuvre un ensemble varié de stratégies de captation du geste (captation directe et indirecte), de technologies de captation ainsi que des techniques audionumériques de traitement de signal et de synthèse. Le choix des solutions trouve sa motivation initiale dans la recherche d'une continuité entre le geste et le résultat sonore, qui donne lieu à une sensation de fluidité dans le vécu corporel du musicien lors $\mathrm{du}$ jeu. L'aller-retour constant entre choix technologiques, programmation et sensation corporelle est fondamental pour ce projet de guitare électrique augmentée. La recherche entreprise sera présentée et développée en détail et profondeur dans une thèse de doctorat dont la soutenance aura lieu en 2010.

\section{BIBLIOGRAPHIE}

Aimi R. M., Hybrid Percussion: Extending Physical Instruments Using Sampled Acoustics, thèse de doctorat, Massachusetts Institute of Technology, 2007.

Laliberté M., "Aux origines des "nouvelles technologies musicales" : virtuosités et archétypes ", Musiques, arts et technologies : pour une approche critique, R. Barbanti, E. Lynch, C. Pardo et M. Solomos (éd.), Paris, l'Harmattan, 2004.

Lähdeoja O., Wanderley M. M., Malloch J., "Instrument augmentation using ancillary gestures for subtle sonic effects", Proceedings of the Sound and Music Computing conference, SMC 2009, Porto, Portugal.

Lähdeoja O., « Guitare électrique augmentée : une approche du contrôle gestuel des "effets" de la guitare électrique ", Actes des journées d'informatique musicale, JIM 2008, Albi, France.

Machover T., "hyperinstruments homepage”, http://www.media.mit.edu/hyperins/

Miranda E. R., Wanderley M. M., New digital musical instruments: control and interaction beyond the keyboard, The computer music and digital audio series, vol. 21, Middleton, Wisconsin, A-R Éditions, 2006.

Refsum-Jensenius A. et al., "Concepts and methods in research on music-related gestures", in Godøy, R. I. and M. Leman (eds.), Musical Gestures. Sound, movement and meaning, London, Routledge, 2009.

Verfaille V., Effets audionumériques adaptatifs, thèse de doctorat à l'université d'Aix-Marseille II, 2003.

Wanderley M. M., Interaction musicien-instrument, application au contrôle gestuel de la synthèse sonore, thèse de doctorat, université Paris 6 / IRCAM, Paris, 2001. 


\section{NOTES}

1. L'utilisation simultanée du signal « direct », des boucles, du microtemps de la granulation, des delays.

2. Effets communs pour la guitare électrique : effets temporels (delay, reverb, chorus, flanger, phaser), la saturation, modulation d'amplitude (tremolo) et modulation en anneau, les filtrages (eq, wah-wah, autofilter), la compression, le bouclage (looping).

INDEX

Mots-clés : guitare électrique, instrument augmenté, rapport geste/son

\section{AUTEUR}

OTSO LÄHDEOJA

CICM, université Paris 8, MSH Paris Nord 